\title{
Carbono orgânico de um Latossolo Vermelho cultivado com cafeeiros em sistemas de manejo agroflorestal e convencional
}

\author{
Franciane Diniz Cogo* \\ Dulcimara Carvalho Nannetti** \\ Davi Lopes do Carmo*** \\ Tales Machado Lacerda**** \\ Alex Nogueira Nannetti*****
}

\section{Resumo}

O conhecimento da influência dos diversos sistemas de manejo do solo com cafeeiro é de grande importância para avaliar a dinâmica de carbono orgânico no solo. Na verificação da qualidade do solo, o carbono orgânico é um dos atributos mais utilizados, pois permite o monitoramento de mudanças de médio a longo prazo. Uma das diferenças marcantes entre os sistemas de manejo é que o cafeeiro sob sistema agroflorestal produz maior quantidade de resíduos orgânicos oriundos das árvores e do próprio sistema de manejo, quando comparado ao manejo convencional, o que pode aumentar os teores e o estoque de carbono orgânico do solo (COS). Este estudo foi realizado com o objetivo de avaliar a densidade do solo e os teores e estoques de COS em cafeeiros nos sistemas de manejo convencional (CC) e agroflorestal (SAF), no Sul de Minas Gerais. Foram coletadas amostras de solo deformadas e indeformadas, nas posições de projeção da copa (PC) e entrelinha (EL) dos cafeeiros e de forma aleatória na mata nativa (MN), na profundidade de $0-20 \mathrm{~cm}$. 0 delineamento experimental adotado foi inteiramente casualizado, com 3 tratamentos e 4 repetições. Os tratamentos foram os seguintes sistemas de manejo: cafeeiro convencional (CC), cafeeiro agroflorestal (SAF) e a mata nativa (MN). O cafeeiro manejado sob sistema agroflorestal apresentou maior teor de COS na projeção da copa e maior estoque de COS quando comparado ao sistema de manejo convencional.

Palavras-chave: Coffea arabica L. Agrofloresta. Estoque de carbono orgânico do solo.

\section{Introdução}

Em função das preocupações ambientais e mudanças climáticas globais, o solo e os sistemas de manejo conservacionistas estão no foco das pesquisas de agricultura que têm em vista conhecer formas de manejo sustentável do solo. Algumas práticas de manejo têm grande influência sobre o teor de carbono orgânico no solo (COS), apresentando potencial de reter carbono atmosférico no solo e contribuir para a mitigação do aquecimento global. Neste sentido, o sistema de manejo alternativo ao preparo convencional na agricultura se torna relevante para o aumento dos teores de cos.

Sistemas de manejo que proporcionam elevados aportes de resíduos vegetais no solo e eficácia no controle de erosão demonstram potencial para sequestrar carbono e contribuir para mitigar o efeito estufa (Hernani et al., 1999; Amado et al., 2001), enquanto que sistemas que possuem o uso indiscriminado de arações e gradagens estimulam a oxidação da matéria orgânica do solo por parte da biomassa microbiana do solo (Lal, 2002).

O balanço de carbono no solo é obtido em função das adições de carbono via fotossíntese pelas plantas e pelas perdas de carbono para a atmosfera mediante a oxidação microbiana do carbono orgânico a $\mathrm{CO}_{2}$, erosão e lixiviação (Buso, Kliemann, 2003; Costa et al., 2008), sendo que o depósito de carbono no solo depende do tipo de vegetação, da fertilidade do solo, dentre outros fatores 
(Batjes, 1999). O processo de decomposição dos resíduos vegetais e da matéria orgânica no solo é proveniente das alterações das taxas metabólicas dos microrganismos, sendo influenciado pelo preparo do solo, manejo de culturas, temperaturas e umidade do solo (La Scala Jr. et al., 2006). Na superfície terrestre, a matéria orgânica constitui o maior reservatório de carbono e a mudança no solo provocada pelas práticas inadequadas de manejo pode levar a uma diminuição no seu estoque de carbono, contribuindo para o aumento das emissões de $\mathrm{CO}_{2}$ na atmosfera (Lal, 1997; Freixo et al., 2002).

O sistema de manejo convencional do solo pode apresentar contribuição no controle de plantas invasoras. No entanto, pode também resultar em declínio dos estoques de cos pelo uso indiscriminado de arações, gradagens e escarificações, com degradação da estrutura do solo e estimulação da oxidação da matéria orgânica do solo por parte da biomassa microbiana deste, levando a intensas perdas de carbono por mineralização e erosão (Castro Filho et al., 1991; Paustian et al., 2000; Lal, 2002). Por outro lado, os sistemas agroflorestais são considerados promissores quanto ao estoque de COS porque a interação das árvores resulta em maior sequestro de $\mathrm{CO}_{2}$ da atmosfera (Benavides et al., 2009; Soto-Pinto et al., 2010), além de não possuir revolvimento de solo e preservar os resíduos vegetais em sua superfície. A eficácia do sistema agroflorestal para armazenar carbono no solo, oriundo da biomassa de árvore, depende da espécie (Kell, 2011), da densidade (Tumwebaze et al., 2011), da textura do solo (Zinn et al., 2011) e de mudanças climáticas globais (Schmidt et al., 2011).

$\mathrm{Na}$ cafeicultura, o sistema agroflorestal abrange $10 \%$ da área mundial, a maior parte pertencente ao Brasil, Guatemala e Peru (Rice, 2011). Este sistema de manejo em cafeeiros proporciona potencial de sequestro de COS porque as árvores contribuem com adição de resíduos orgânicos (Youkhana e Idol, 2011). Trabalhos realizados com cafeeiros sob sistemas consorciados com Eucalyptus deglupta, Erythrna poeppigiana e Albizia adianthifolia mostraram valores de estoques de COS superiores, quando comparado, ao monocultivo de cafeeiro em pleno sol (Ávila, 2001; Dossa et al., 2008).

Diante da importância da atividade cafeeira e dos seus impactos ambientais, este estudo objetivou avaliar os teores e estoques de carbono orgânico em solos com cafeeiros sob sistemas de manejo convencional e agroflorestal, no Sul de Minas Gerais.

\section{Material e métodos}

\subsection{Localização da área experimental}

O estudo foi realizado no município de Machado, no Sul do Estado de Minas Gerais, nas proximidades das coordenadas geográficas $21^{\circ} 39^{\prime} 59^{\prime \prime} \mathrm{S}$ e $45^{\circ} 55^{\prime} 16^{\prime \prime} \mathrm{W}$. O solo é um Latossolo Vermelho com textura franca (273 $\mathrm{g} \mathrm{kg}^{-1}$ de argila, $459 \mathrm{~g} \mathrm{~kg}^{-1}$ de areia e $268 \mathrm{~g} \mathrm{~kg}^{-1}$ de silte). 0 clima é do tipo Köppen (CWa), com temperatura média anual de $21,0{ }^{\circ} \mathrm{C}$ e a média pluviométrica anual é de $1.824 \mathrm{~mm}$ (Marques, 2003).

\subsection{Caracterização da lavoura cafeeira}

A lavoura de café em sistema convencional (CC) foi implantada em 1989, no espaçamento 4,0 x 1,0 m, com a cultivar Mundo Novo. Para o plantio foram feitas covas para evitar o revolvimento do solo. O solo foi mantido sempre coberto de plantas invasoras manejadas com a aplicação de herbicida de pós-emergência, duas vezes ao ano e com o uso de roçadoura costal motorizada, aproximadamente cinco vezes ao ano. Todos os tratos culturais foram realizados manualmente e as adubações minerais e a calagem foram realizadas conforme as recomendações técnicas (Guimarães et al., 1999), com o uso de formulações de NPK (20-05-20) divididas em três aplicações no período de dezembro a março, de acordo com a análise química do solo e adubação foliar de micronutrientes de acordo com a análise foliar.

A lavoura de café em sistema agroflorestal (CAF) foi implantada em 1985, com a variedade Mundo Novo, no espaçamento de 3,5 x 1,0 m e em 1998 foi adotado o modelo de produção natural orgânico em sistema agroflorestal consorciado com frutíferas e árvores nativas. Bananeiras e eucaliptos 
cultivados ao redor dos talhões de café servem como quebra-vento. As árvores nativas, frutíferas e plantas anuais se apresentam espalhadas irregularmente entre as linhas dos cafeeiros. Desde 1997, a fertilização do solo e a nutrição do cafeeiro são resultantes da aplicação dos resíduos orgânicos e vegetais, tais como palha de café e a serrapilheira originada do próprio sistema. A mata nativa (MN) foi utilizada para fins de comparação, por se tratar de um sistema em seu estado natural e sem histórico de atividade antrópica.

\subsection{Coletas e análises de amostras de solo}

Foram coletadas amostras de solo deformadas e indeformadas na profundidade $0-20 \mathrm{~cm}$, sendo que as indeformadas foram coletadas com auxílio de um amostrador de Uhland utilizando anéis volumétricos de alumínio e deformadas com trado holandês. Para os cafeeiros, as amostras foram coletadas nas posições de projeção da copa (PC) e entrelinha (EL); as amostras foram coletadas aleatoriamente na mata nativa. $\mathrm{O}$ delineamento experimental adotado foi inteiramente casualizado, com 3 tratamentos e 4 repetições. A caracterização química das áreas estudadas é apresentada na Tabela 1.

Tabela 1. Caracterização química do solo na projeção da copa para os cafeeiros e na mata nativa, profundidade de $0-20 \mathrm{~cm}$.

\begin{tabular}{|c|c|c|c|c|c|c|c|c|c|c|}
\hline \multirow{2}{*}{ Tratamentos } & \multirow{2}{*}{$\begin{array}{c}\mathrm{pH} \\
\text { água }\end{array}$} & $P$ & $\mathrm{~K}^{+}$ & $\mathrm{Ca}^{+2}$ & $\mathrm{Mg}^{+2}$ & SB & CTC & $\mathrm{H}+\mathrm{Al}$ & $\mathrm{Al}^{+3}$ & \multirow{2}{*}{$\begin{array}{l}\mathrm{V} \\
\%\end{array}$} \\
\hline & & \multicolumn{2}{|c|}{$\mathrm{mg} \mathrm{dm}^{-3}$} & \multicolumn{6}{|c|}{$\mathrm{cmol}_{\mathrm{c}} \mathrm{dm}^{-3}$} & \\
\hline CC & 4,99 & 13,2 & 105,7 & 1,73 & 0,75 & 2,75 & 8,75 & 6,00 & 0,50 & 34,2 \\
\hline SAF & 5,68 & 6,43 & 116,0 & 4,08 & 1,15 & 5,53 & 9,33 & 3,81 & 0,00 & 58,6 \\
\hline $\mathrm{MN}$ & 5,61 & 3,73 & 299 & 4,48 & 1,50 & 6,75 & 10,7 & 4,04 & 0,00 & 61,7 \\
\hline
\end{tabular}

CC: convencional; SAF: agroflorestal; MN: mata nativa.

Fonte: elaboração própria.

As análises de COS foram realizadas em amostras deformadas, sendo o teor COS determinado pela oxidação úmida (EMBRAPA, 1997). A densidade do solo foi determinada pelo método do cilindro de Uhland (Blake e Hartge, 1986). Os estoques de COS foram calculados para cada tratamento, na profundidade $0-20 \mathrm{~cm}$, pela equação 1 :

$C$ total $=\left(\frac{T C \cdot D S \cdot E S C S}{10}\right)$

Em que:

C total = estoque de $\mathrm{C}$ total $\left(\mathrm{Mg} \cdot \mathrm{ha}^{-1}\right)$;

$\mathrm{TC}=$ teor de carbono total $\left(\mathrm{g} \mathrm{kg}^{-1}\right)$;

DS = densidade do solo $\left(\mathrm{g} \mathrm{cm}^{-3}\right)$;

ESCS = espessura da camada de solo $(\mathrm{cm})$.

A densidade do solo (Ds) utilizada para o cálculo foi obtida pela razão entre a massa da amostra seca a $105^{\circ} \mathrm{C}$ e o volume do cilindro, conforme as equações 2 e 3 :

$D s=\frac{m}{V_{c}}$

Em que:

Ds = densidade do solo $\left(\mathrm{g} \mathrm{cm}^{-3}\right)$;

$\mathrm{m}=$ massa de solo seco $(\mathrm{g})$;

$\mathrm{Vc}=$ volume do anel $\left(\mathrm{cm}^{3}\right)$. 
$V c=\left(\frac{\pi d^{2}}{4}\right) \cdot h c$

Em que:

$\mathrm{Vc}=$ volume do anel $\left(\mathrm{cm}^{3}\right)$;

$\mathrm{d}=$ diâmetro do anel $(\mathrm{cm})$;

$\mathrm{hc}=$ altura do anel $(\mathrm{cm})$.

\subsection{Análises estatísticas}

As análises de variância foram realizadas no programa estatístico JMP 5.1 (SAS Institute, Cary, NC). Para o teste de médias, foi utilizado o teste de Tukey, para $\mathrm{P}<0,05$ entre os tratamentos. Para verificar a diferença entre as posições de amostragem linha e entrelinha, foi utilizado o teste de $t$, para $\mathrm{P}<0,05$. Para a validação da análise de variância, foram aplicados os testes O’Brien, para a normalidade, e de Bartlett, para a homocedasticidade, que demostrou que as hipóteses são satisfatórias. Em seguida, procedeu-se o teste $F$, que indicou que houve significância, e as médias foram comparadas por meio do teste de Tukey, a $5 \%$.

\section{Resultados e Discussão}

Os valores de densidade do solo não apresentaram diferença significativa $(\mathrm{P}=0.2726)$ entre os sistemas de manejo quando comparados à mata nativa (Tabela 2). A adoção de práticas de conservação do solo por meio do manejo de plantas invasoras e também pelo não revolvimento do solo, desde o início da implantação do cafeeiro convencional, contribuíram para não apresentar alteração neste atributo. Estes resultados diferem dos resultados encontrados por Carvalho et al. (2004), que encontraram menor densidade do solo em sistema agroflorestal quando comparado ao sistema de manejo convencional.

Tabela 2. Valores de densidade do solo $\left(\mathrm{g} \mathrm{cm}^{-3}\right)$ para os três tratamentos em diferentes posições de amostragem.

\begin{tabular}{ccc}
\hline \multirow{2}{*}{ Tratamentos } & \multicolumn{3}{c}{ Posições de amostragem } \\
\cline { 2 - 3 } & PC & EL \\
\hline CC & 1,23 & 1,25 \\
SAF & 1,26 & 1,27 \\
MN & 1,30 & 1,30 \\
\hline
\end{tabular}

CC: convencional; SAF: agroflorestal; MN: mata nativa; PC: projeção da copa; EL: entrelinhas.

Fonte: elaboração própria.

Os teores de COS não apresentaram diferença significativa entre as posições (projeção da copa e entrelinhas do cafeeiro) dentro de cada sistema de manejo com cafeeiros (convencional e agroflorestal), enquanto que, entre os sistemas de manejo com cafeeiros, houve na projeção da copa (Figura 1). O cafeeiro agroflorestal apresentou valores de COS significativamente maiores para a posição de projeção da copa quando comparado ao cafeeiro manejado em sistema convencional, sendo o sistema de manejo decisivo para a adição ou perda do COS. Certamente, o maior teor de COS encontrado no sistema de manejo agroflorestal se deve ao maior volume de resíduos orgânicos produzidos pelas árvores e adubação orgânica realizada com palha de café, além de adubação verde nas entrelinhas. Este manejo, quando combinado com o não revolvimento do solo, contribui para a estabilização dos agregados e eleva os teores de matéria orgânica por meio de exsudados e rápida formação, morte e decomposição de raízes finas mediante a ciclagem bioquímica. Os resultados ressaltam a importância do alto aporte de resíduos vegetais quando se visa ao acúmulo de COS (Lovato et al., 2004; Bayer et al., 2006). Em um estudo realizado por Campanha et al. (2007), mostrou- 
se que o acúmulo de resíduos orgânicos em sistemas agroflorestais foi superior àquele em sistema convencional, com acréscimo significativo de matéria seca na serrapilheira.

O cafeeiro em sistema de manejo convencional resultou em baixa capacidade de aporte de carbono orgânico, porque possivelmente a adição anual foi inferior à quantidade perdida por oxidação da matéria orgânica pela microbiota do solo, com diminuição dos teores de COS ao longo do tempo. As operações de práticas agrícolas neste manejo, com a ação da temperatura e umidade do solo, influenciam a taxa de emissão de carbono na forma de $\mathrm{CO}_{2}$ para a atmosfera (Franzluebbers et al., 1995; Kessavalou et al., 1998).

Figura 1. Teores carbono orgânico no solo (COS) em função dos sistemas de manejo com cafeeiros (CC: convencional e SAF: agroflorestal) e (MN: mata nativa).

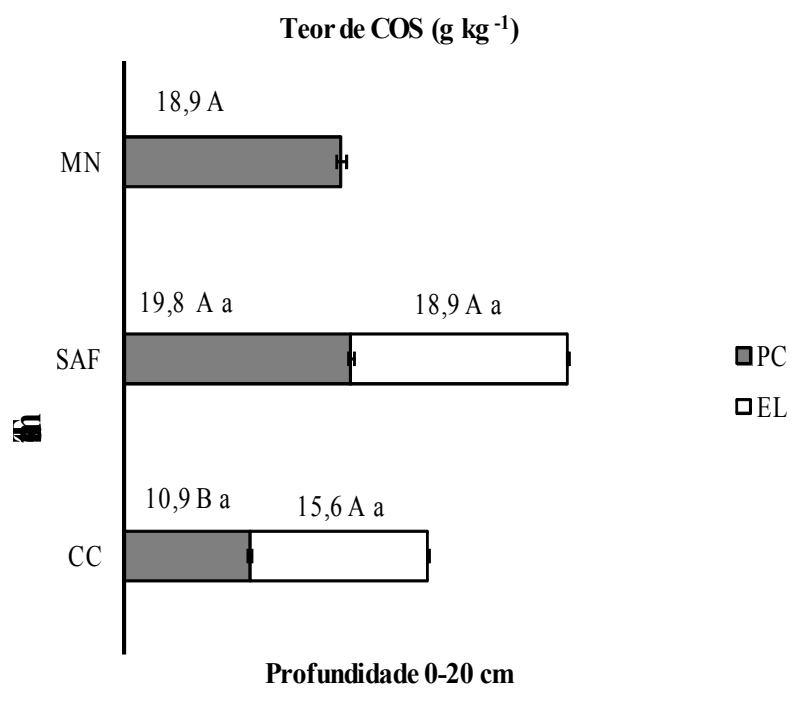

PC: projeção da copa; EL: entrelinhas. As médias seguidas da mesma letra maiúsculas na vertical não diferem entre si pelo teste de Tukey e letra minúscula na horizontal não difere entre si pelo teste de $t$, a $5 \%$ de probabilidade.

Fonte: elaboração própria.

Os estoques de COS apresentaram diferença significativa entre os tratamentos (Figura 2), com valor significativamente maior para o cafeeiro agroflorestal em comparação com o cafeeiro convencional. De acordo com Houghton et al. (1991), o declínio no estoque de carbono orgânico do solo ocorre quando se faz a conversão de florestas nativas em sistemas agrícolas, entretanto, neste trabalho o sistema agroflorestal não apresentou diferença quando comparado à mata nativa. Este resultado se deve à maior biomassa produzida pelo cafeeiro sob sistema agroflorestal, indicando que o uso do solo neste sistema apresenta sequestro de carbono. Em um trabalho realizado por Nogueira et al. (2009), mostrou-se que os sistemas agroflorestais proporcionaram maiores estoque de COS quando comparados aos sistemas convencionais.

A redução do estoque de COS para o cafeeiro convencional pode ser justificado por reduzir o máximo à vegetação nas entrelinhas de plantio, por meio do uso de herbicida para o controle das plantas invasoras, aumento da erosão do solo, aos processos mais acelerados de mineralização da matéria orgânica e oxidação de carbono orgânico do solo e às menores quantidades de aportes orgânicos em sistemas manejados comparativamente a sistemas agroflorestais e matas nativas.

Figura 2. Estoque de carbono orgânico no solo (COS) em função dos sistemas de manejo com cafeeiros (CC: convencional e SAF: agroflorestal) e MN: mata nativa. 


\section{Estoque de COS (Mg.ha-1)}

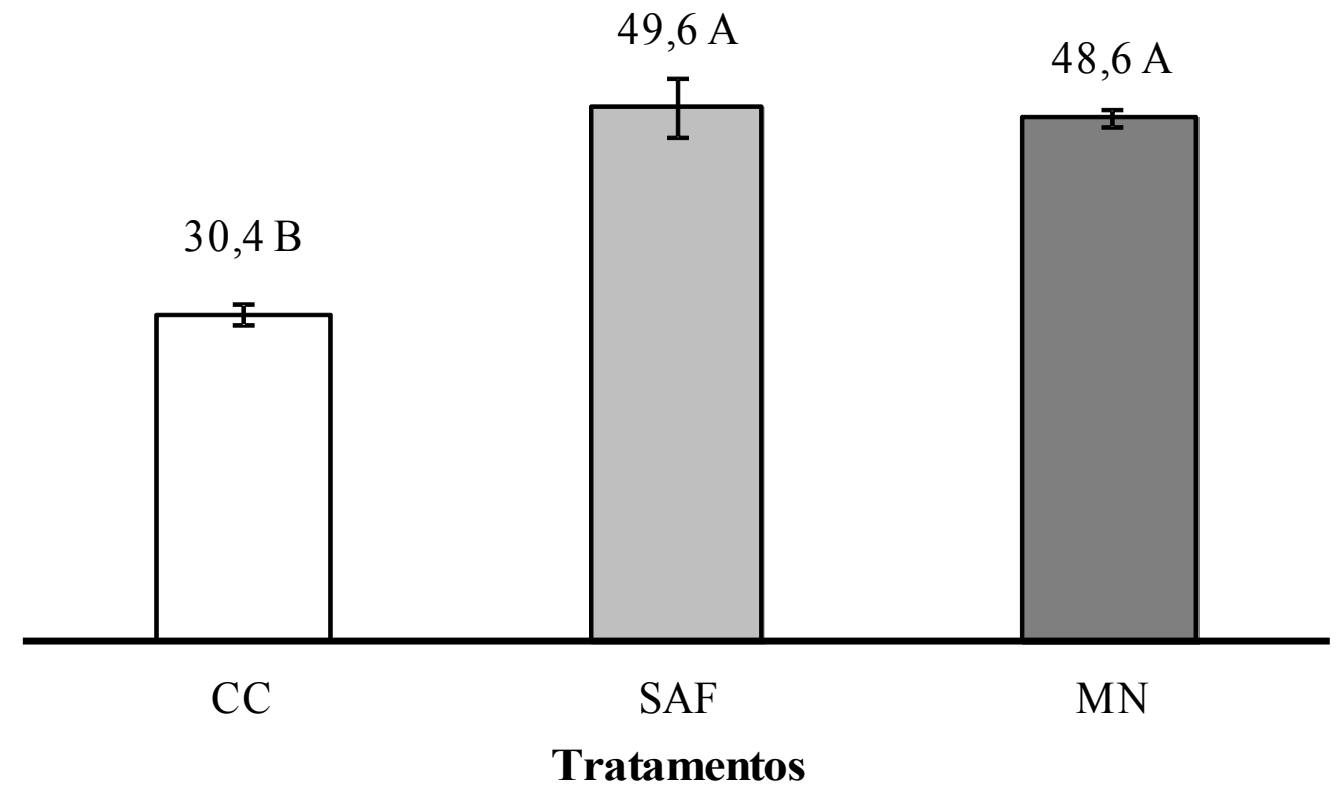

Médias seguidas pela mesma letra não diferenciam entre si pelo teste de Tukey, a $5 \%$ de probabilidade. Fonte: elaboração própria.

\section{Conclusões}

1. Os valores de densidade do solo e de teores de COS não apresentaram diferença entre as posições de projeção da copa e entrelinha, dentro de cada sistema de manejo com cafeeiros agroflorestal e convencional.

2. Os teores de COS no cafeeiro sob sistema convencional foi menor em relação ao cafeeiro sob sistema agroflorestal na posição de projeção da copa.

3. Em relação à mata nativa, verificou-se que o cafeeiro em sistema agroflorestal apresentou estoques de COS semelhante, enquanto que o cafeeiro em sistema convencional apresentou estoque menor.

\section{Organic carbon of a red Latosol in coffee management systems agroforestry and conventional}

\section{Abstract}

Knowledge of the influence of different soil management systems with coffee is of great importance to evaluate the dynamics of soil organic carbon. In checking the quality of soil organic carbon is one of the attributes most commonly used because it allows monitoring of changes in the medium to long term. Managed under the coffee agroforestry system differs from conventional production and the largest addition of organic residues derived from the trees and the management system itself, which can promote increased levels of stock and soil organic carbon (SOC). This study was conducted 
to evaluate the levels and stocks of SOC in coffee plants in conventional tillage systems (CC) and agroforestry (SAF), in southern Minas Gerais. We collected soil samples and undisturbed, the positions of the canopy projection (CP) and leading (EL) of the trees and random in native forest (NF) at a depth of $0-20 \mathrm{~cm}$. The experimental design was completely randomized with three treatments and four replications. The treatments consisted of the following management systems: conventional coffee (CC), coffee agroforestry (SAF) and the native forest (NF). The coffee managed under agroforestry exhibited higher levels of COS in the top projection and a larger stock of COS when compared to conventional management system.

Key-words: Coffea arabica L. Agroforestry. Soil organic carbon stock.

* Mestranda em Ciência do Solo pela Universidade Federal de Lavras-MG (DCS/UFLA). E-mail: fdcogo@yahoo.com.br

** Professora do IFSULDEMINAS, campus Machado, rodovia Machado-Paraguaçu, km 07, CEP 37.750-000. E-mail:

dulcimara@mch.ifsuldeminas.edu.br

*** Doutorando em Ciência do Solo pela Universidade Federal de Lavras-MG (DCS/UFLA). E-mail: davigoldan@yahoo.com.br

**** Tecnólogo em Cafeicultura pelo IFSULDEMINAS, campus Machado-MG. E-mail: tales_lacerda@yahoo.com.br

***** Engenheiro Agrônomo - Mestrando em Produção na Agropecuária - UNIFENAS-MG. E-mail: alexnannetti@axtelecom.com.br

\section{Referências Bibliográficas}

AMADO, T. J. C.; BAYER, C.; ELTZ, F. L. F.; BRUM, A. C. R. Potencial de culturas de cobertura em acumular carbono e nitrogênio no solo no plantio direto e a melhoria da qualidade ambiental. Brasileira de Ciência do Solo, Viçosa, v.25, p.189-197, 2001.

ÁVILA, G. F.; JIMNEZ, F.; BEER, J.; MEZ, G. M.; IBRAHIM, M. Almacenamiento, fijación de carbono y valoración de servicios ambientales em sistemas agroforestales em Costa Rica. Agroforestales América, v.30, p.32-41, 2001.

BATJES, N. H. Management options for reducing $\mathrm{CO}_{2}$-concentrations in the atmosphere by increasingcarbon sequestration in the soil. Report 410-200-031, Dutch National Research Programme on Global Air Pollution and Climate Change \& Technical Paper 30, International Soil Reference and Information Centre, Wageningen, 1999.

BAYER, C.; MARTIN-NETO, L.; MIELNICZUK, J.; PAVINATO, A.; DIECKOW, J. Carbon sequestration in two Brazilian Cerrado soils under no-till. Soil and Tillage Research, v.86, p.237-245, 2006.

BENAVIDES, C. M.; GONZÁLEZ, I. C. C.; HURTADO, H.; SOTO, E. V. Cuantificación de carbono en la biomasa aerea de café(Coffea arábica L.) con sombra, en la comarca palo de sombrero, Jinotega, Nicaragua. La calera, Managua, v.12, n.6, 2009.

BLAKE, G. R., HARTGE, K. H. Bulk density. In: KLUTE, A, ed. Methods of soil analysis. Physical and mineralogical methods. Madison: ASA, 1986. p.363-375.

BUSO, W. H. D.; KLIEMANN, H. J. Relações de carbono orgânico e de nitrogênio total e potencialmente mineralizável com o nitrogênio absorvido pelo milheto. Pesquisa Agropecuária Tropical, v.33, p.97-105, 2003.

CAMPANHA, M. M.; SANTOS, R. H. S. S; FREITAS, G. B.; MARTINEZ, H. E. P.; GARCIA, S. L. R.; FINGER, F. L. Análise comparativa das características da serrapilheira e do solo em cafezais (Coffea arabica L.) cultivados em sistema agroflorestal e em monocultura, na Zona da Mata MG. Revista Árvore, v.31, p.805-812, 2007.

CASTRO FILHO, C.; VIEIRA, M. J. CASÃO Jr., R. Tillage methods and soil and water conservation in southern Brazil. Soil and Tillage Research, v.20, p.271-283, 1991. 
CARVALHO, R.; GOEDERT, W. J.; ARMANDO, M. S. Atributos físicos da qualidade de um solo sob sistema agroflorestal. Pesquisa Agropecuária Brasília, v.39, p.1153-1155, 2004.

COSTA, F. S.; BAYER, C.; ZANATTA, J. A.; MIELNICZUK, J. Estoque de carbono orgânico no solo e emissões de dióxido de carbono influenciadas por sistemas de manejo no sul do brasil. Brasileira de Ciência do Solo, v.32, p.323-332, 2008.

GUIMARÃES, P. T. G.; GARCIA, A. W. R.; ALVARES V., V. H. A.; PREZOTTI, L. C.; VIANA, A. S.; MIGUEL, A. E.; MALAVOLTA, E.; CORRÊA, J. B.; LOPES, A. S.; NOGUEIRA, F. D.; MONTEIRO, A. V. C.; OLIVEIRA, J. A. In: RIBEIRO, A.C.; GUIMARÃES, P. T. G.; ALVAREZ-VENEGAS, V. H. Recomendações para o uso de corretivos e fertilizantes em Minas Gerais: $5^{a}$ aproximação. Viçosa: CFSEMG, 1999. p. 289-302.

DOSSA, E. L.; FERNANDES, E. C. M.; REID, W. S.; EZUI, K. Above- and belowground biomass, nutrient and carbon stocks contrasting an open-grown and a shaded coffee plantation. Agroforest Systems, v.7, p.103-115, 2008.

EMPRESA BRASILEIRA DE PESQUISA AGROPECUÁRIA. Manual de métodos de análise de solo. 2ed. Rio de Janeiro: Centro Nacional de Pesquisa de Solos, 1997. 212 p.

FRANZLUEBBERS, A. J.; HONS, F. M.; ZUBERER, D. A. Tillage-induced seasonal changes in soil physical properties affecting soil $\mathrm{CO} 2$ evolution under intensive cropping. Soil and Tillage Research, v.34, p.41-60, 1995.

FREIXO, A. A.; MACHADO, P. L. O. A.; GUIMARÃES, C. M.; SILVA, C. A.; FADIGAS, F. S. Estoques de carbono e nitrogênio e distribuição de frações orgânicas de Latossolo do cerrado sob diferentes sistemas de cultivo. Brasileira Brasileira de Ciência do Solo, v.26, p.425-434, 2002.

HERNANI, L. C.; KURIHARA, C. H.; SILVA, W. M. Sistemas de manejo de solo e perdas de nutrientes e matéria orgânica por erosão. Brasileira Brasileira de Ciência do Solo, v.23, p.145-154, 1999.

HOUGHTON, R.A.; SKOLE. D.L.; LEFKOWITZ, D.S. Changes in the landscape of Latin America between 1850 and 1985. II Net release of $\mathrm{CO}_{2}$ to the atmosphere. Forest Ecology Management, v.38, p.173-199, 1991.

KELL, D. K. Breeding crop plants with deep roots: their role in sustainable carbon, nutrient and water sequestration. Annals of Botany, v.108, p. 407-418, 2011.

KESSAVALOU, A.; DORAN, J. W.; MOSIER, A. R.; DRIJBER, R. A. Greenhouse gas fluxes following tillage and wetting in a wheat-fallow cropping system. Journal of Environmental, v.27, p.1105-1116, 1998.

LAL, R. Soil carbon dynamic in cropland and rangeland. Environmental Pollutinn, v.116, p.353-362, 2002.

LAL, R. Residue management, conservation tillage and soil restoration for mitigating greenhouse effect by $\mathrm{CO}_{2}$-enrichment. Soil and Tillage Research, v.43, p.81-107, 1997.

LA SCALA Jr., N.; LOPES, A.; PANOSSO, A. R.; CAMARA, F. T.; PEREIRA, G. T. Soil $\mathrm{CO}_{2}$ efflux following Rotary tillage of a tropical soil. Soil and Tillage Research, v.84, p.233-235, 2005. 
LOVATO, T.; MIELNICZUK, J.; BAYER, C. VEZZANI, F. Adições de carbono e nitrogênio e sua relação com os estoques no solo e com o rendimento do milho em sistemas de manejo. Brasileira de Ciência do Solo, v.28, p.175-187, 2004.

MARQUES, H. S. Uso de geotecnologias no estudo das relações entre solos, orientação de vertentes e o comportamento espectral de áreas cafeeiras em Machado, Minas Gerais. Lavras, Universidade Federal de Lavras, 2003. 82p. (Tese de Mestrado)

NOGUEIRA, R. S.; OLIVEIRA, T. S.; TEIXEIRA, A. S.; CAMPANHA, M. M. Distribuição Espacial do Estoque de Carbono Orgânico Total em Luvissolos sob Sistemas Agrícolas Convencionais e Agroflorestais. Revista Brasileira de Agroecologia, v.4, p.2118-2122, 2009.

PAUSTIAN, K.; SIX, J.; ELLIOTT, E. T.; HUNT, H. W. Management options for reducing $\mathrm{CO}_{2}$ emissions from agricultural soils. Biogeochemistry, v.48, p.147-163, 2000.

RICE, A. R. Fruits from shade trees in coffee: how important are they? Agroforest Systems, v.83, p. 41-49, 2011.

SAS Institute 2003. JMP, Statistics and Graphics Guide, Version 5.1, SAS Inc., Cary, NC, 593 pp.

SCHMIDT, M. W. I.; S. TORN, M. S.; ABIVENT.; S.; DITTMAR, T.; GUGGENBERGER, G. Persistence of soil organic matter as an ecosystem property. Nature, London, v.478, n.15, p.49-56, 2011.

SOTO-PINTO, L.; ANZUETO, M.; MENDOZA, J.; FERRER, G. J.; JONG, B. Carbon sequestration through agroforestry in indigenous communities of Chiapas, Mexico. Agroforest Systms, v.78, p.39-51, 2010.

TUMWEBAZE, B. S.; BEVILACQUA, E.; BRIGGS, R..; VOLK, T. Soil organic carbon under a linear simultaneous in Uganda. Agroforest System, v.80, p.1-13, 2011.

YOUKHANA, A.; IDOL, T. Addition of Leucaena-KX2 mulch in a shaded coffee agroforestry system increases both stable and labile soil C fractions. Soil Biology \& Biochemistry, v. 43, p. 961-966, 2011.

ZINN, Y. L.; LAL. R.; DIMAS, V. S.; RESCK, D. V. S. Eucalypt plantation effects on organic carbon and aggregation of three different-textured soils in Brazil. Soil Research, v.49, p.614-624, 2011. 